\title{
Semianalytic treatment for propagation in finite photonic crystal waveguides
}

\author{
L. C. Botten, A. A. Asatryan, and T. N. Langtry
}

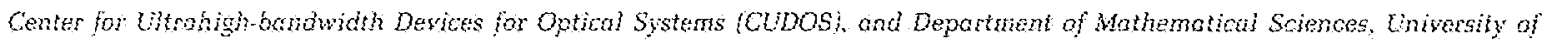

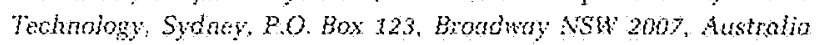

T. P. White, C. Martijn de Sterke, and R. C. McPhedran

Canter for Uthaingh-bundwidth Devices for Ophical Systems (CUDOS). Sohod of Physics, University of Sydney, Sydney, NSW 2006. Anstriatio

Recoivad Nowonber 12.2002

We present a semianalytic theory for the properties of iwo dimensional photonic erystal wavegudes uf finte lerugh. For singlemode guides, the transmission spectrum and fielo intensity can be acurately doscribed by a simple swo-parameter model. Analogies are drawn with Fabry-perot interferometers, and stenteralized

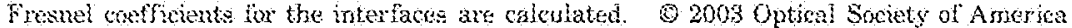
OCIS coles: $2307370,250,5900,0501950,0502230$.

Photonic crystals ${ }^{1,2}$ (PCs) are thought to be key buxiding blocks for future micro-optical technology in that they provide a versatile means to control hight. Wavegudes based on $\mathrm{PC}_{5}$, with their capacity to guide light without diffraction losses, are likely to become integral components in all-optical processors. Mekis et al. showed theoretically that it is possible to bend light by $90^{\circ}$ without significant loss, a result that was later confirmed experimentally. Interconnections such as $Y$ junctions, "T junctions, and chamel-drop filters have also been proposed. It is thus vital to understand the mechanism of guiding and coupling for such devices.

The coupling problem is challenging ${ }^{3}$ and general, involving the interfacing of one guide to another (e.g., at a bend or junction) or to an extemal medium. Previous treatments ${ }^{7}$ considered coupling in and out separately. Here, we address the full circuit problem-coupling in, propagation, and coupling out-the solution of which, until now, has relied almost exclusively on computational techniques. Although these methods are accurate, they are computationally intensive and exploit little of the underlying physics. Here, we present a semianalytic method to calculate the held profiles and the transmission of straight PC waveguides based on Bloch modes and their reflection and transmission at interfaces by use of generalized Fresnel coeffieients.

Motivating this work is a desire for a simple method of calculating the properties of a straight pC waveguide formed in a two-dimensional finite PC. Figure $1(a)$ shows the TM field intensity in a cluster comprising a square symmetric lattice (spacing i) of cyinders and a channel of width $d$, generated by a point source near the waveguide entrance. The resuits, computed wath a multipole meibod, exbibit resonant behavior associated with a superposition of forwaed-and backward-propagating fields, similar to the resonances of a Fabry Perot $\left(\mathrm{F}^{3}\right)$ interferometer. These resonances can aiso be clearly seen in the transmission spectrum (dashed curve) in Fig. 2(a), up to the modal cutoff at $\lambda / d=3.496$. Using the FP analogy, we describe the propagation constants and characterize the transmissivity with an exact generalization of the Airy formulation" for a FP interferometer, leading to a simple two-parameter model for a single-mode guide.

The key step in generalizing the Airy formulas is the computation of the relevant Bloch modes. The strueture is modeled by a finite stack [see Fig. $1(b)$ ] of $L$ grating layers, each of thickness $d$ and with a periodic defect (width $d$ ) that is due to the removal of a cylinder, generating a lattice with a single channel per supercall. Numerical investigations using the multipole method have shown that coupling between the guides is negligible for separations exceeding $10 d$, and thus we model the waveguide array [Fig. $1(a)$ ], using supercell period $D=11 d$.

The diffraction properties of each grating are described by plane-wave reflection and transmission

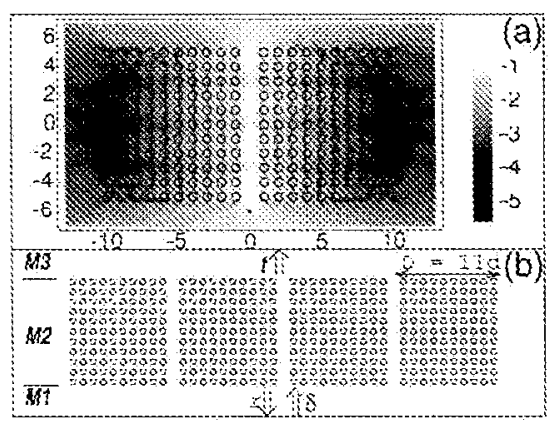

Fig. 1. (a) Fichd intensity (decinial log scale) in a finte duster due to a point source fblack dot. The waveguide corresponds to the removal of a line of cylinders of radius $a / d=0.3$, refractive index $y_{e}=3$ (in air $y_{b}=1$ ), and wave length $\lambda / d=3.19$. (b) Supercell model apponoximating geometry $(a)$, with gudes spaced by $D=11 d$. 


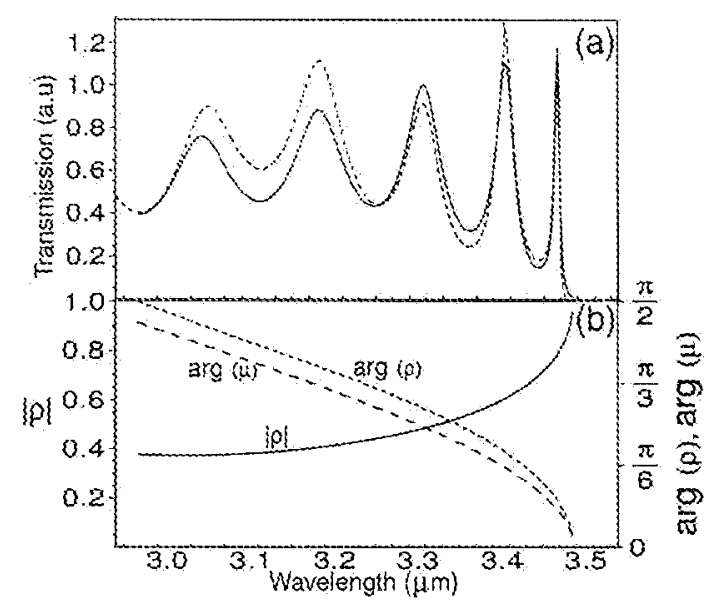

Fig. 2. (a) Transmission spectrum calculated with multipole (dashed curve) and asymptotic (solid curve) models. (b) ho (solid curve), arg $p$ (short-dashed curves, and ang $p$ (long-dashed curve) veretss wavelength. The left (right) axis displays the magnitude argument?.

scattering matrices." Between consecutive grating layers the modes are described by plane-wave expansions comprising forward- (upward in Fig. 1) and backwari- (downward-) propagating components, respectively represented by vectors of coefficients $f$. and $\mathbf{f}$, where the entries correspond to the amplitudes in the various orders. The Bloch modes $\tilde{f}=\left[f_{+}{ }^{T} f_{-}\right]^{T}$ are then computed with a transfer matrix method, ${ }^{11}$ in which the eigenvalue problem $T \hat{\mathrm{f}}=\mu \hat{f}$ for the interlayer transiation operator $T$ is solved to yield the modes $\tilde{f}$. The set of all modes, derived from the solution of an eigenvalue problem. can be partitioned into forward- and backwardpropagating states." For a rectangular latice, it cax be shown that if $\mu=\exp \left(i \beta_{0} d\right)$ is an eigenvalue of the forward state $\left(f_{+}, f_{-}\right)$, in which $\beta_{0}$ is the Bloch constant corresponding here to the mode's propagation constant in the guide, the paired backward state has eigenvalue $\mu^{-1}$ and eigenvector $\left(f_{-} f_{+}\right)$. Collecting all forward and backward eigenstates, we form miatrices $F_{2}$ from the column vectors $f_{ \pm}$ and a diagonal matrix $A$ from the eigenvalues $\mu$. Modes are sequenced with propagating states listed first.

Within medium $M 2$ of the structure $\{\mathrm{Fig} .1(\mathrm{~b})\}$, the fields are expanded in the Bloch basis of forward $\left\{A,\left(F_{+}, F_{-}\right)\right\}$and backward $\left\{A^{-1},\left(F_{-}, F_{+}\right\}\right.$states. In free space, media M1 and M3 of Fig. 16?, we expand fields in the plane-wave basis. In $\mathrm{Mn}$, there is a forward-incident field $\hat{\delta}$ and a backward-reflected field $x$, whereas in Mo there is only a forwardtransmitted field $t$. The components of these vectors correspond again to the plane-wave orders of the field expansion. The incident field launches forward Bloch waves that are reflected from the rear (23) interface to generate backward modes. The matching of forward and backward components at the two interfaces of the L-layer crystal keats to the solution of the field problem.
In this formulation it is convenient and intuitive to separate the propagation of Bioch waves from theix scattering at the interfaces; the latter is characterized by generalized Fresnel reflection and transmission $c 0-$ efficients. This separation treatrment leads to the vector of transmitted field amplitudes given by $t=\mathrm{T} \delta$, where $T$ is the matrix

$$
\mathbf{T}=\mathbf{T}_{23} \Lambda^{L}\left(\mathbf{I}-\mathbf{R}_{23} \Lambda^{L} \mathbf{R}_{23} \Lambda^{L}\right)^{-1} \mathbf{T}_{12}
$$

One can understand the structure of Eq. (1), the matrix analog of the scalar Airy transmission formula, by reading from right to left with the field transmited (T) from $\mathrm{ML}$ to $\mathrm{M2}$, propagated $\left(\mathrm{A}^{L}\right)$ through $\mathrm{M2}$, and transmitted $\left(\mathrm{T}_{23}\right.$ ) from $\mathrm{M} 2$ to $\mathrm{M} 3$, with resmant propagation and reflection between the front and rear boundaries represented by $\left(\mathbf{I}-\mathbf{R}_{2.2} \mathbf{\Lambda}^{L} \mathbf{R}_{23} \mathbf{\Lambda}^{L}\right)^{-1}$. Explicit expressions for the reflection $\left(R_{i j}\right)$ and transmission $\left(T_{i}\right)$ coefficients are found by expression of fields as linear combinations of forward-and backwardpropagating Bloch modes and plane waves (in the erystal and free space) and matching their forward and backward plane-wave components at theix common interface. In this way we show that $\boldsymbol{R}_{21}$ and $\mathbf{R}_{23}$, the crystal-air reflection coefficients, are given by $\mathbf{R}_{21}=\mathbf{R}_{23}=-\boldsymbol{\rho}_{*}$ with $\boldsymbol{\rho}_{*}=\mathbf{F}_{+}^{-1} \mathbf{F}_{-}{ }^{13}$. The matrix $\mathbf{T}_{\mathrm{Z}}=\mathbf{F}_{\mathrm{H}}\left(\mathbf{I}-\boldsymbol{\mu}_{*}{ }^{2}\right)$ denotes the crystal-air transmission coefficient and is derived from the sum $\left(F_{-}-F_{-}, p_{-}\right)$of two terms, the forward components of the forward modes $\left(\boldsymbol{F}_{+}\right)$, and the forward components of the backward modes $\left.F_{-}\right)$that are generated by a reflection $\left(-\rho_{\infty}\right)$ at the rear interface. The calculation of the air-crystal transmission coefficient $\mathbf{T}_{12}$ involves only forwand-propagating modes and yields the sim. pler form $T_{12}=F_{+}^{-1}$, which is merely a change of basis from plarie waves to Bloch modes. Substituting the Fresnel coefficients into Eq. (1) then yields the matrix form of the Airy transmission formula ${ }^{9}$ for a symmetric $\mathrm{EP}$ inter ferometer:

$$
\mathbf{T}=\mathbf{F}_{+}\left(\mathbf{I}-\boldsymbol{\rho}_{+}^{2}\right) \mathbf{\Lambda}^{L}\left(\mathbf{I}-\boldsymbol{\beta}_{x} \Lambda^{L} \boldsymbol{\mu}_{\times} \boldsymbol{\Lambda}^{L}\right)^{-1} \mathbf{F}_{+}^{-1}
$$

Equation (2) is exact, in that including all modes yields a result identical to that generated by the recursive computation of $\mathbf{T}$ from the scattering matrices of the individual gratings. However, a major simplification is possible for suitably long guides. The Bloch basis, used to expand the fields, includes rapidly decaying evanescent terms. A though for an infinitely long guide the energy carried by these modes is precisely zero, this is not true for finite guides. However, for guides longer than a few layers, the evanescent field decay lets us ignore their contribution, and only the propagating Bloch modes need be considered. For a single-mode guide, Eq. (2) may be simplified by use of the Sherman-Morrison formula ${ }^{12}$ for the inversion of a matrix with a rank one perturbation caused by $\Lambda^{3}$ as $L \rightarrow \infty$. Together with modal orthogonality relations this formula greatly simplifies the expression for the transmitted flux to yield

$$
\varepsilon_{2}=\frac{1-|\rho|^{2}}{\left.\mid 1-\rho^{2} \mu^{2 L}\right\}^{2}}|\boldsymbol{\delta}+\delta|^{2}
$$




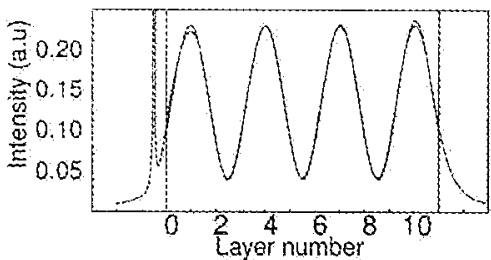

Fig. 3. Comparison of the field intensity [Fig. 1(a)] along the waveguide axis, calculated with the multipole method (dashed curve) and the asymptotic model (solid curve).

Here, $\mu$ is the eigenvalue of the single propagating mode, and $p$, the $(1,1)$ element of $\rho_{c}$, is its complex Fresnel reflection coefficient. The denominator of Eq. (3) characterizes the resonance, and the numerator $\left(1-|\rho|^{2}\right)$ expresses the net energy propagation of a forward mode of unit flux and a backward mode of flux $|\rho|^{2}$ generated by reflection at the rear surface. The coupling of the incident field into the primary Bloch mode is given by an overlap integral $v_{+} \delta$, where $\mathbf{v}_{\dot{ }}$ denotes row 1 of $\mathbf{F}_{+}^{-1}$. Figure $2(b)$ shows $|p|$, $\arg (\beta)$, and $\arg (\mu)=\beta_{0} d$ over wavelengths between the band edge of the bulk crystal and the guide cutoff, where $\rho \rightarrow 1$ and $\mu \rightarrow 1$. Modal cutoff corresponds to the edge of a full bandgap of the composite guide-crystal, at which the structure behaves as a perfect reflector $\{|\rho|=1)$.

In Fig. 2(a), the approximate result (3), indicated by the solid curve, is compared with the full multipole calculation for the finite guide of Fig. 1(a). The multipole results were obtained by integration of the total flux across the rear of the crystal. Even though Eq. (3) is for a periodic array of waveguides and neglects evanescent fields, it predicts accurately the positions of the transmission peaks and provides good agreement with the transmittance at longer wavelengths. The interference extrema are determined almost exclusively by parameters $\mu$ and $p$, and the magnitude of the transmittance is scaled by the coupling coefficient $\left|v_{+} \delta\right|^{2}$. The source of the discrepancy between the model and the multipole calculation arises from the difficulty in estimating the transmittance in a finite structure by evaluating the flux integral over a finite surface.

The utility and accuracy of the two-parameter $(\mu, p)$ model are further illustrated by the midchannel intensity plot of Fig. 3 . The dashed curve is from the multipole calculation for a guide of length $L=11 d$, whose geometry is identical to that used for Fig. $1(a)$, and the solid curve is for the asymptotic model, the spatial dependence of which can be shown to be proportional to $\left[\mu^{n}-\rho \mu^{2 L-n}\right\}^{2}$ at the interface to layer $n$. There is good agreement in both the fringe location and. the fringe visibility, $V=2|\rho| /\left(1+|\rho|^{2}\right)$, with deviations arising only at the front surface, where evarescent modes are required for full characterization of the field.

In conclusion, we have developed an elegant treatment for PC waveguides through a generalization of the Fresnel reflection and transmission coefficients. For single-mode propagation, the guide can be characterized fully by only two parameters, providing accurate predictions of transmittance and fields within the guide. The ideas here can be gerieralized to morecomplex coupling problems such as propagation at bends and joints and will lead to new tools to aid in the design of $\mathrm{PCs}$.

The support of the Australian Research Council for the Center for Ultrahigh-bandwidth Devices for Optical Systems (CUDOS) is acknowledged. L. C. Botten's e-mail address is lindsay.botten@uts.edu.au.

\section{References}

1. J. D. Joamopoulos, R. D. Maede, and J. N. Winn, Photonic Crystals (Princeton U. Press, Princeton, N.d., 1995).

2. C. M. Soukoulis, ed, Photonic Crystals and Light Localization in the 21st Century, Vol. 563 of NATO ASI Series C: Mathematical and Physics Sciences (Kluwer Academic, Dordrecht, The Netherlands, 2001).

3. A. Mekis, J. C. Chen, I. Kuriand, S. Fan, P. R. Villeneuve, and J. D. Joannopoulos, Phys. Rev. Lett. 77, 3787 (1996)

4. S.Y. Lin, E. Chow, V. Hietala, P. P. Villeneuve, and J. D. Joannopoulos, Science 282, 274 (1998).

5. J. Yoneliura, M. Ikeda, and T. Baba, J. Lightwave Technol. 17,1500 (1999).

6. J. D. Joannopoulos, S. Fan, A. Mekis, and S. G. Johnson, in Photonic Crystals and Light Localization in the 21st Century, C. M. Sonkoulis, ed., Vol. 563 of NATO ASI Series C: Mathematical and Physics Sciences (Kluwer Academic, Dordrecht, The Netherlands, 2001), pp. 1-24.

7. S. Fan, P. R. Villeneuve, J. D. Joannopoulous, and H. A. Haus, Phys. Rev. Lett. 80, 960 (1998).

8. A. A. Asatryan, K. Busch, R. C. McPhedran, L. C. Botten, C. Martijn de Sterke, and N. A. Nicorovici, Phys. Rev. E 63, 046612 (2001).

9. M. Born and E. Wolf, Principles of Optics, 6th (corrected) ed. (Pergamon, Oxford, 1993), Sec. 1.6.4.

10. L. C. Botten, N. A. Nicorovici, A. A. Asatryan, R. C. McPhedran, C. Martijn de Sterke, and P. A. Robinson, J. Opt. Soc. Am. A 17, 2165 (2000).

11. L. C. Botten, N. A. Nicorovici, K. C. McPhedran, C. Martijn de Sterke, and A. A. Asatryan, Phys. Rev. D 64, 046603 (2001).

12. W. H. Press, S. A. Teukolsky, W. T. Vetterling, and B. P. Flannery, Numerical Recipes in Fortran 77 (Cambridge U. Press, Cambridge, 1997). 
THOMSON

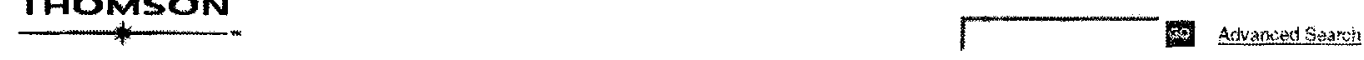

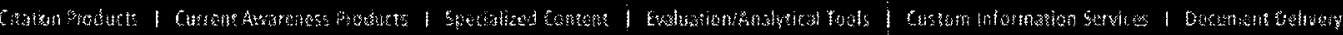

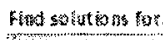

Geaventic

GoverHath

now wheert.

Konnonkt

\section{moours \\ cowernancs byo weter}

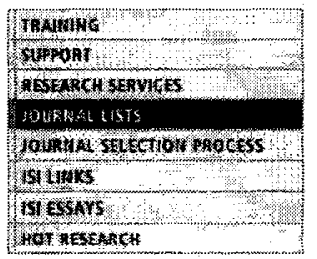

gegrons

$\sqrt{\text { Business Website }}$
SEATCH RESULTS

ISI Master Joumal List

Search lems: 0.46\%9592

Total ioumals founc: 1

The followito tite(s) mathed your recuest

Joumats $1+1$ (ot) 1 \&

FOAMAY FO FRIN:

OPHCS LETHES

Semimonthly

SSN: 0140.9592

OPTCAL SOO AMER, 2OYOMASSACHUSETSS AVE NW. WASHNGTON, USA, DO. 20026

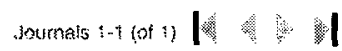

